\title{
DETERMINAÇÃO QUANTITATIVA DE TNT E HNS POR TG E FT-IR
}

\section{Gilson da Silva*}

Divisão de Química I, Diretoria de Patentes, Instituto Nacional da Propriedade Industrial, Rua Mayrink Veiga, 9, $20090-210$ Rio de Janeiro - RJ, Brasil

\section{Elizabeth da Costa Mattos}

Divisão de Sistemas de Defesa, Instituto de Aeronáutica e Espaço, Comando-Geral de Tecnologia Aeroespacial, Praça Marechal do Ar Eduardo Gomes, 50, 12228-904 São José dos Campos - SP, Brasil

\section{Rita de Cássia Lazzarini Dutra e Milton Faria Diniz}

Divisão de Química, Instituto de Aeronáutica e Espaço, Comando-Geral de Tecnologia Aeroespacial, Praça Marechal do Ar Eduardo Gomes, 50, 12228-904 São José dos Campos - SP, Brasil

\section{Koshun Iha}

Instituto Tecnológico de Aeronáutica, Comando-Geral de Tecnologia Aeroespacial, Praça Marechal do Ar Eduardo Gomes, 50, 12228-904 São José dos Campos - SP, Brasil

\begin{abstract}
QUANTITATIVE DETERMINATION OF TNT AND HNS BY TG AND FT-IR. 2,4,6-trinitrotoluene (TNT) is an energetic material that shows scarce crystalline properties that can be improved by addition of $2,2^{\prime}, 4,4^{\prime}, 6,6^{\prime}$-hexanitrostilbene (HNS) in the crystallization process. HNS is a very important high explosive used in a variety of military, aerospace and industrial formulations owing to its suitable properties. It is an insensitive and thermal stable explosive that can be produced from 2,4,6-trinitrotoluene (TNT). The purpose of this work is the quantitative determination of HNS and TNT in explosives by thermogravimetric analysis (TG) and Fourier transform infrared spectroscopy (FT-IR).
\end{abstract}

Keywords: TNT; HNS; quantification.

\section{INTRODUÇÃO}

O 2,4,6-trinitrotolueno (TNT) é um material energético muito utilizado para fins militares e civis. ${ }^{1}$ A substância pura apresenta fusão a $80,8{ }^{\circ} \mathrm{C}$. Durante um longo período, a temperatura de fusão foi o critério mais utilizado para a determinação do teor de pureza do TNT, pois está relacionada com a presença de outros constituintes no material (isômeros). Atualmente, têm-se reconhecido que este fator não é a resposta de todas as dificuldades do uso prático deste explosivo, que apresenta uma série de problemas, tais como baixa temperatura de fusão, propriedades mecânicas pobres (formação de cavidades e trincas no TNT moldado) e exudação (migração para a parte externa) de vários componentes eutéticos (misturas isoméricas de trinitrotoluenos e dinitrotoluenos). ${ }^{2}$

A presença de cavidades e trincas no TNT moldado gera o risco de uma detonação prematura, pois aumenta a área superficial do corpo, ampliando sua superfície passível de combustão. ${ }^{3}$ Por outro lado, a exudação, fenômeno que ocorre com maior incidência no verão, causa problemas de segurança quando a migração de componentes explosivos atinge peças rosqueáveis das ogivas, podendo ocasionar detonação acidental durante o manuseio do material. ${ }^{3}$

Uma série de nitroderivados pode ser obtida a partir do TNT. Um dos exemplos é a obtenção do 2,2',4,4',6,6'-hexanitroestilbeno (HNS), que pode ser conduzida por meio da oxidação do TNT com hipoclorito de metal alcalino. ${ }^{1}$

Durante a extração de petróleo, certos explosivos são utilizados para perfurar o solo rochoso. Tanto pela profundidade dos poços, quanto pelo conseqüente aumento da temperatura ambiente, explosivos resistentes ao calor estão apresentando maior interesse para

\footnotetext{
*e-mail: gilsondasilva@uol.com.br
}

a indústria do petróleo. ${ }^{4,5}$ Muitos desses explosivos apresentam iniciação em temperaturas superiores a $260^{\circ} \mathrm{C}$, podendo permanecer em temperaturas elevadas por algumas horas sem apresentar decomposição térmica. ${ }^{4,6}$

O 2,2',4,4',6,6' -hexanitroestilbeno ou 1,2 bis $((2,4,6$ trinitrofenil)-etileno), HNS, é um exemplo comum desse grupo de explosivos resistentes ao calor. É um composto nitro aromático com baixa pressão de vapor, alta estabilidade química a vácuo e temperaturas de fusão relatadas entre 316 e $321^{\circ} \mathrm{C}$, apresentando ainda decomposição em temperaturas superiores a $260{ }^{\circ} \mathrm{C},{ }^{4,7}$ como requerido para o emprego na exploração de petróleo. A Figura 1 ilustra a estrutura da molécula de HNS.<smiles>O=[N+]([O-])c1cc([N+](=O)[O-])c(C=Cc2c([N+](=O)[O-])cc([N+](=O)[O-])cc2[N+](=O)[O-])c([N+](=O)[O-])c1</smiles>

Figura 1. Estrutura molecular do 2,2',4,4',6,6'-hexanitroestilbeno (HNS)

O HNS é empregado na confecção de detonadores ${ }^{8}$ cordéis de detonação ${ }^{9}$ e reforçadores, booster. Reforçadores são materiais mais energéticos utilizados em um "trem explosivo" para a iniciação de explosivos de alta potência. ${ }^{10}$ Em um "trem explosivo", diversos explosivos são colocados em contato de forma que o mais sensível (explosivo primário), iniciado por meio térmico, elétrico, etc., inicie um segundo explosivo (reforçador) esse, por sua vez, transmite a detonação a um terceiro explosivo de grande potência (explosivo secundário). ${ }^{11}$ 
O HNS é ainda muito utilizado em sistemas aeroespaciais, militares e industriais. É escolhido para essa finalidade por inúmeras razões: apresenta baixa sensibilidade, não entrando em decomposição quando exposto a variações ambientais, como descargas elétricas, impactos, atrito ou temperaturas elevadas. De fato, a única forma de iniciação deste explosivo é por meio de onda de choque. ${ }^{12} \mathrm{~A}$ Tabela 1 apresenta algumas propriedades físico-químicas do HNS. ${ }^{6,13,14}$

Tabela 1. Propriedades fisico-químicas do HNS

\begin{tabular}{lc}
\hline Propriedades & \\
\hline Fórmula empírica & $\mathrm{C}_{14} \mathrm{H}_{6} \mathrm{~N}_{6} \mathrm{O}_{12}$ \\
Massa molar & $450,1 \mathrm{~g} / \mathrm{mol}$ \\
Volume de gás na explosão & $766 \mathrm{dm}^{3} / \mathrm{kg}$ \\
Calor de explosão ( $\mathrm{H}_{2} \mathrm{O}$ liq.) & $4089,7 \mathrm{~J} / \mathrm{g}$ \\
Calor de explosão ( $\mathrm{H}_{2} \mathrm{O}$ gas.) & $4010,2 \mathrm{~J} / \mathrm{g}$ \\
Massa específica & $1,74 \mathrm{~g} / \mathrm{cm}^{3}$ \\
Temperatura de fusão & $318^{\circ} \mathrm{C}$ \\
Sensibilidade ao impacto & $5 \mathrm{Nm}$ \\
Sensibilidade ao atrito & $240 \mathrm{~N}$ \\
\hline
\end{tabular}

Em processo onde o TNT necessita ser recristalizado, sua nucleação pode ser orientada por meio da adição do HNS ao mesmo. ${ }^{15}$

As técnicas de análise térmica vêm sendo amplamente utilizadas no estudo da decomposição de materiais energéticos. ${ }^{16}$ A maior vantagem em relação às outras técnicas é a utilização de somente alguns miligramas da amostra. Estas técnicas podem ser utilizadas tanto na caracterização quanto no desenvolvimento de materiais energéticos, pois muitas informações sobre um explosivo podem ser obtidas antes de uma quantidade perigosa ser preparada. ${ }^{17}$

Outras técnicas podem ser usadas na identificação, caracterização e quantificação de explosivos. A espectroscopia na região do infravermelho (IR) permite a confirmação dos produtos, seja por comparação entre as bandas de absorção entre um produto e sua referência, ou por comparação com as bandas de absorção de referência do material encontradas na literatura. ${ }^{6}$

Este trabalho apresenta metodologias para a quantificação de composições contendo TNT e HNS, por meio de termogravimetria (TG) e espectroscopia de infravermelho com transformada de Fourier (FT-IR). A metodologia desenvolvida para quantificação dos explosivos por ambas as técnicas visa determinar um procedimento mais rápido e de menor custo com relação às análises por HPLC, uma vez que, além de caros os solventes necessários aos ensaios cromatográficos, o preparo das amostras é realizado com dimetilsulfóxido (DMSO), que é tóxico e prejudica a filtração da amostra devido à alta viscosidade.

\section{PARTE EXPERIMENTAL}

Os ensaios termogravimétricos foram realizados em um equipamento TG/SDTA Mettler (Modelo TGA/SDTA851e). O equipamento foi calibrado na razão de aquecimento utilizada, $10^{\circ} \mathrm{C} / \mathrm{min}$, com alumínio e índio. Foram utilizadas cerca de $1,0 \mathrm{mg}$ de amostra em cada ensaio realizado e a decomposição do material foi analisada no intervalo de temperatura entre 50 e $650^{\circ} \mathrm{C}$ em cadinhos de alumina $\left(\mathrm{Al}_{2} \mathrm{O}_{3}\right)$ sob vazão de nitrogênio gasoso de $40 \mathrm{~mL} / \mathrm{min}$. Os resultados representam uma média de três ensaios. ${ }^{18}$

As análises espectrométricas foram realizadas em um espectrômetro FT-IR Spectrum 2000 PerkinElmer, região de 4000 a $400 \mathrm{~cm}^{-1}$, resolução $4 \mathrm{~cm}^{-1}$, ganho $1 \mathrm{e} 40$ varreduras; e em um espectrômetro FT-IR Spectrum One PerkinElmer, região de 4000 a
$400 \mathrm{~cm}^{-1}$, resolução $4 \mathrm{~cm}^{-1}$, ganho $1 \mathrm{e} 40$ varreduras . As amostras foram analisadas segundo a técnica de transmissão, pastilha de $\mathrm{KBr}$ na proporção 0,8:400 mg. Os resultados representam uma mediana de cinco ensaios. ${ }^{18}$

A cromatografia a líquido de alta performance (HPLC) foi utilizada para a determinação dos teores reais dos explosivos nas composições estudadas. As análises cromatográficas foram feitas num cromatógrafo líquido da Waters 7725I Breeze, com sistema de bombeamento isocrático modelo 1515 , detector UV de duplo canal modelo 2487, injetor manual da Rheodyne com loop de $10 \mu \mathrm{L}$. Os dados foram processados por meio do software Breeze versão 3.30. Foi utilizada a coluna de fase reversa $\mu$ Bondapak C18 $(3,9 \times 300$ $\mathrm{mm}$ ) e comprimento de onda de $254 \mathrm{~nm}$. As soluções dos explosivos utilizadas nos ensaios HPLC foram preparadas com DMSO. Os resultados representam uma média de três ensaios. ${ }^{18}$

Foram preparadas, em laboratório, composições padrão contendo HNS e TNT, que foram cuidadosamente pulverizadas num almofariz. Essas composições foram analisadas por HPLC onde foram determinados os teores reais de cada explosivo nas mesmas. O procedimento adotado visou a redução de erros referentes à perda de material durante a etapa de mistura e pulverização das composições.

\section{RESULTADOS E DISCUSSÃO}

\section{Análise termogravimétrica}

As curvas de decomposição térmica dos ensaios realizados com cada composição padrão (Std.) contendo TNT e HNS são apresentadas na Figura 2. A composição onde apenas TNT estava presente (Std. 1) apresentou uma única etapa de decomposição, que ocorreu em temperaturas inferiores a $280^{\circ} \mathrm{C}$. Em composições contendo TNT e HNS foi observado um segundo patamar de decomposição após $280^{\circ} \mathrm{C}$.

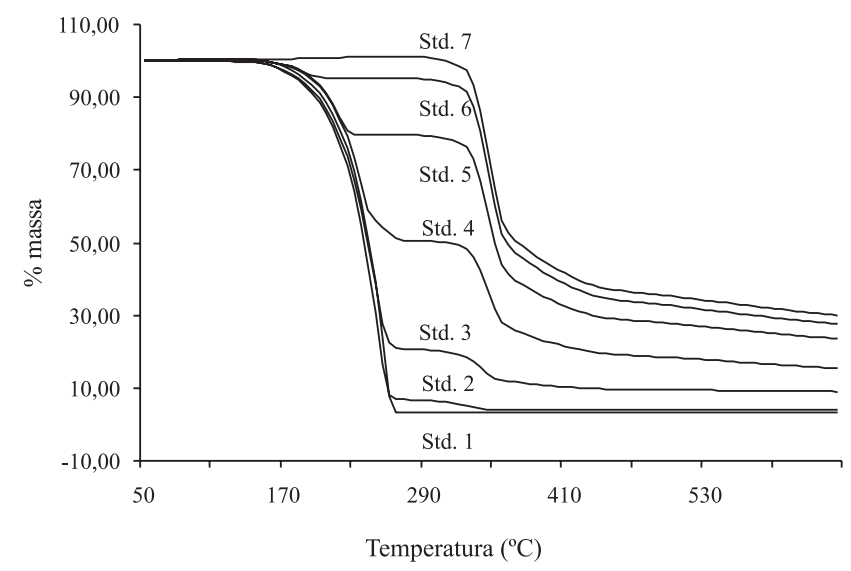

Figura 2. Curvas TG (decomposição térmica de composições padrão de HNS e TNT) obtidas na razão de aquecimento de $10^{\circ} \mathrm{C} /$ min e sob atmosfera dinâmica de $40 \mathrm{~mL} / \mathrm{min}$ de $\mathrm{N}_{2}$

A Tabela 2 apresenta os teores de HNS e TNT presentes em cada uma das composições padrão analisadas, bem como o percentual de decomposição a $280^{\circ} \mathrm{C}$.

Por meio da Figura 2, e dados da Tabela 2, é possível observar que em quaisquer das composições onde os dois explosivos estavam presentes ocorreu a formação de dois patamares distintos de decomposição, bem como que quanto maior a concentração de HNS na composição, maior é o resíduo final apresentado à $650^{\circ} \mathrm{C}$, Figura 2. É possível observar ainda que o calor decorrente da decomposição 
Tabela 2. Teores de HNS e TNT presentes nas composições padrão e percentual de decomposição das composições à $280^{\circ} \mathrm{C}$

\begin{tabular}{lccc}
\hline Std. & $\%$ TNT & $\%$ HNS & Perda de massa a $280^{\circ} \mathrm{C}(\Delta \mathrm{m} \%)$ \\
\hline 1 & 100 & 0 & 97,15 \\
2 & 95,16 & 4,84 & 93,13 \\
3 & 81,26 & 18,74 & 79,77 \\
4 & 50,92 & 49,08 & 49,57 \\
5 & 22,27 & 77,73 & 20,66 \\
6 & 5,55 & 94,45 & 5,03 \\
7 & 0 & 100 & 0 \\
\hline
\end{tabular}

do TNT não é suficiente para a iniciação do HNS, que continua apresentando decomposição acima de $280{ }^{\circ} \mathrm{C}$ mesmo em misturas com TNT.

Um gráfico relacionando os teores de HNS presente em cada composição com perda de massa $(\Delta \mathrm{m} \%)$ a $280{ }^{\circ} \mathrm{C}$ pode ser observado na Figura 3.

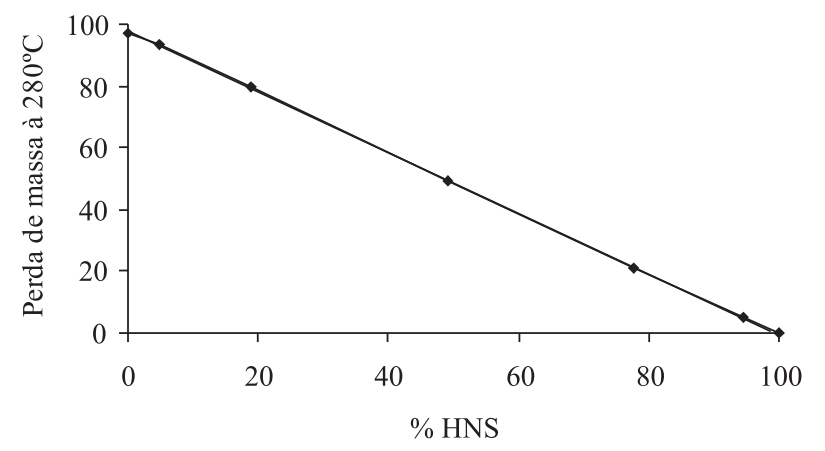

Figura 3. Gráfico de correlação entre os teores de HNS presentes nas composições analisadas e a perda de massa a $280{ }^{\circ} \mathrm{C}$

A reta média obtida por regressão linear apresentou ótimo coeficiente de determinação $\left(\mathrm{R}^{2}\right), 0,9999$, e é representada pela Equação 1:

$y=-0,9808 x+97,645$

A Equação 1 foi utilizada para calcular o teor de HNS presente em três composições preparadas previamente, às quais os teores de HNS foram também analisados por HPLC, visando verificar a eficiência da metodologia de quantificação de HNS em misturas com TNT por meio da termogravimetria. A Figura 4 apresenta a decomposição térmica das composições cujos teores dos explosivos foram quantificados por meio de TG.

Na Figura 4 é possível verificar a presença dos dois explosivos, HNS e TNT, em virtude dos dois patamares observados na decomposição térmica de cada composição. A Tabela 3 apresenta a decomposição e os teores dos explosivos presentes em cada composição. Os teores de explosivos foram calculados por meio da Equação 1 e são comparados, na Tabela 3, com os teores reais, obtidos por HPLC, onde o desvio entre os valores obtidos por ambas as técnicas de quantificação empregadas é apresentado.

É possível verificar, por meio da Tabela 3, que quanto maior for o teor de HNS na composição menor é o erro que ocorre entre as técnicas de quantificação. Ao retornar às curvas originais das análises TG efetuadas para cada composição e cujos percentuais de decomposições são apresentados na Tabela 4, pode-se verificar que a variação no percentual de decomposição da amostra B em cada análise, em

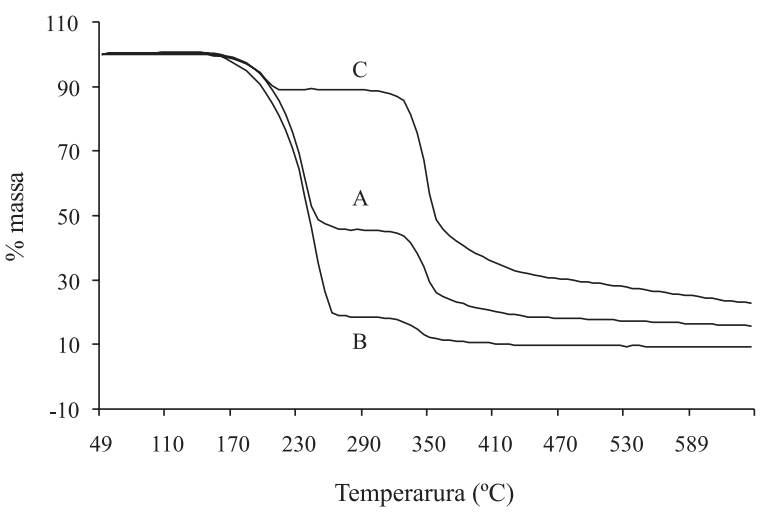

Figura 4. Curvas TG (decomposição térmica de composições problema de HNS e TNT) obtidas na razão de aquecimento de $10^{\circ} \mathrm{C} / \mathrm{min}$ e sob atmosfera dinâmica de $40 \mathrm{~mL} / \mathrm{min}$ de $\mathrm{N}_{2}$

Tabela 3. Teores de HNS quantificados por TG e comparados com os valores reais determinados por HPLC

\begin{tabular}{lcccc}
\hline Amostra & $\Delta \mathrm{m} \%$ a $280^{\circ} \mathrm{C}$ (média) & $\% \mathrm{HNS}_{\mathrm{TG}}$ & $\% \mathrm{HNS}_{\mathrm{HPLC}}$ & $\% \mathrm{E}$ \\
\hline A & 54,93 & 43,55 & 44,19 & 1,4 \\
$\mathrm{~B}$ & 81,99 & 15,96 & 15,06 & 6,0 \\
$\mathrm{C}$ & 11,02 & 88,32 & 89,47 & 1,3 \\
\hline
\end{tabular}

relação às outras composições, é mais elevado. Esse fato pode estar relacionado com a evaporação de gases nitrosos do TNT, ${ }^{1}$ cuja fusão ocorre em $80,7^{\circ} \mathrm{C},{ }^{15}$ que tornam as curvas térmicas menos estáveis ou mesmo à falta de homogeneidade na amostra.

Tabela 4. Teores de HNS e TNT quantificados por TG em cada rampa de aquecimento das amostras

\begin{tabular}{lccc}
\hline Amostras & $\Delta \mathrm{m} \% \mathrm{a} 280^{\circ} \mathrm{C}$ & $\Delta \mathrm{m}(\%)$ médio & Desvio \\
\hline \multirow{4}{*}{ A } & 55,29 & & \\
& 54,63 & 54,9 & 0,3 \\
& 54,86 & & \\
B & 81,59 & & \\
& 83,51 & 82,0 & 1,4 \\
& 80,87 & & \\
C & 11,69 & & \\
& 9,98 & 11,0 & 0,9 \\
\hline
\end{tabular}

A quantificação de HNS em composições contendo HNS e TNT por meio da termogravimetria mostrou grande eficiência.

\section{Análise por FT-IR}

Segundo a literatura, ${ }^{19}$ quando se possui um número pequeno de valores experimentais os valores dos parâmetros valor médio, média aritmética e desvio padrão determinados por métodos estatísticos clássicos estão sujeitos a grandes erros, difíceis de serem avaliados, devido a distribuição não uniforme dos erros aleatórios nas séries de dados. Em espectroscopia estes erros podem ser causados por flutuações instantâneas na temperatura, dimensões geométricas, tensão de rede elétrica, quantidades elétricas nos componentes eletrônicos, deslocamento aleatório dos elementos, entre outros fatores. Então se recomenda uma avaliação diferente, empregando na determinação do 
valor médio a mediana em vez da média aritmética; ou seja, o valor de elemento do meio da série de dados arranjados de acordo com sua magnitude se seu número é impar ou a média dos dois valores medianos, se seu número é par. 2:

Assim, no cálculo do desvio médio padrão $\left(\sigma_{\mu}\right)$ usa-se a Equação

$\sigma_{\mu}=\sigma / \sqrt{ } \mathrm{n}$

onde: $\sigma_{\mu}=$ desvio médio padrão; $\mu=$ valor mediano de absorvância das alíquotas, no caso das pastilhas analisadas para cada amostra; $\sigma=$ desvio padrão $=\mathrm{K}_{\mathrm{R}} \mathrm{R} ; \mathrm{K}_{\mathrm{R}}=$ coeficiente para cálculo do desvio médio padrão para a faixa de variação. Para 5 medidas, $K_{R}=0,430$; $\mathrm{R}$ = faixa de variação (maior valor - menor valor de absorvância das alíquotas analisadas para cada amostra); $\mathrm{n}=$ número de medidas, ou seja, número de alíquotas analisadas para cada amostra.

Para cálculo do desvio relativo (em percentagem) usa-se a Equação 3:

Desvio relativo $(\%)=\left(\sigma_{\mu} / \mu\right) 100$

Observando as fórmulas estruturais, apresentadas na Figura 5, verifica-se que, em termos estruturais, TNT e HNS são muito parecidos uma vez que basicamente são constituídos das mesmas ligações químicas. Ambos os componentes são aromáticos e contêm grupos nitro. Os grupos metila da molécula de TNT se fundem, durante o processo de síntese de HNS, dando origem a um grupo olefínico trans do HNS $(-\mathrm{CH}=\mathrm{CH}-){ }^{18}$<smiles>Cc1c([N+](=O)[O-])cc([N+](=O)[O-])cc1[N+](=O)[O-]</smiles>

(TNT)<smiles>O=[N+]([O-])c1cc([N+](=O)[O-])c(/C=C/c2c([N+](=O)[O-])cc([N+](=O)[O-])cc2[N+](=O)[O-])c([N+](=O)[O-])c1</smiles>

(HNS)
Figura 5. Fórmulas estruturais de TNT e HNS

Devido a esta semelhança estrutural seus espectros de absorção na região do infravermelho são muito semelhantes, como apresentado na Figura 6. As principais absorções características do TNT estão em torno de 3097 e $3057 \mathrm{~cm}^{-1}$, estiramento (v) de grupos $\mathrm{CH}$ aromático; 1617 e $1602 \mathrm{~cm}^{-1}, \mathrm{vC}=\mathrm{C}$ aromático; 1538 e $1353 \mathrm{~cm}^{-1}$, $v \mathrm{ArNO}_{2}, 908,792,735,719$ e $704 \mathrm{~cm}^{-1}$, deformação $(\delta)$ de grupos $\mathrm{CH}$ aromático. ${ }^{18}$

Já as principais absorções características de HNS estão em trono de $3100 \mathrm{~cm}^{-1}$, estiramento (v) de grupos $\mathrm{CH}$ aromático; 1613 e 1603 $\mathrm{cm}^{-1}, \mathrm{vC}=\mathrm{C}$ aromático e olefínico; 1540 e $1350 \mathrm{~cm}^{-1}, \mathrm{ArNO}_{2} ; 957$ $\mathrm{cm}^{-1}$, vibração $(\omega)$ de grupos $\mathrm{CH}=\mathrm{CH}$ trans; 740,737 e $717 \mathrm{~cm}^{-1}$, deformação $(\delta)$ de grupos $\mathrm{CH}$ aromáticos. ${ }^{18}$

$\mathrm{A}$ absorção devido à deformação tipo vibração $\omega \mathrm{CH}=\mathrm{CH}$ trans característica do HNS, apesar de sua média intensidade, está razoavelmente isolada, banda assinalada na Figura 6; foi a absorção que melhor se apresentou para a quantificação de HNS em presença de TNT, sendo adotada como banda analítica. Para efetuar os cálculos necessários na elaboração da curva de calibração foram adotadas as linhas bases 1769 e $836 \mathrm{~cm}^{-1}$.

As composições padrão com teores variados de HNS e TNT, como apresentados na Tabela 2, foram analisadas em quintuplicata por pastilhas com brometo de potássio $(0,8: 400 \mathrm{mg})$, para a construção da curva de calibração. As demais composições (A, B e C) também foram analisadas por meio da mesma técnica, onde os teores dos

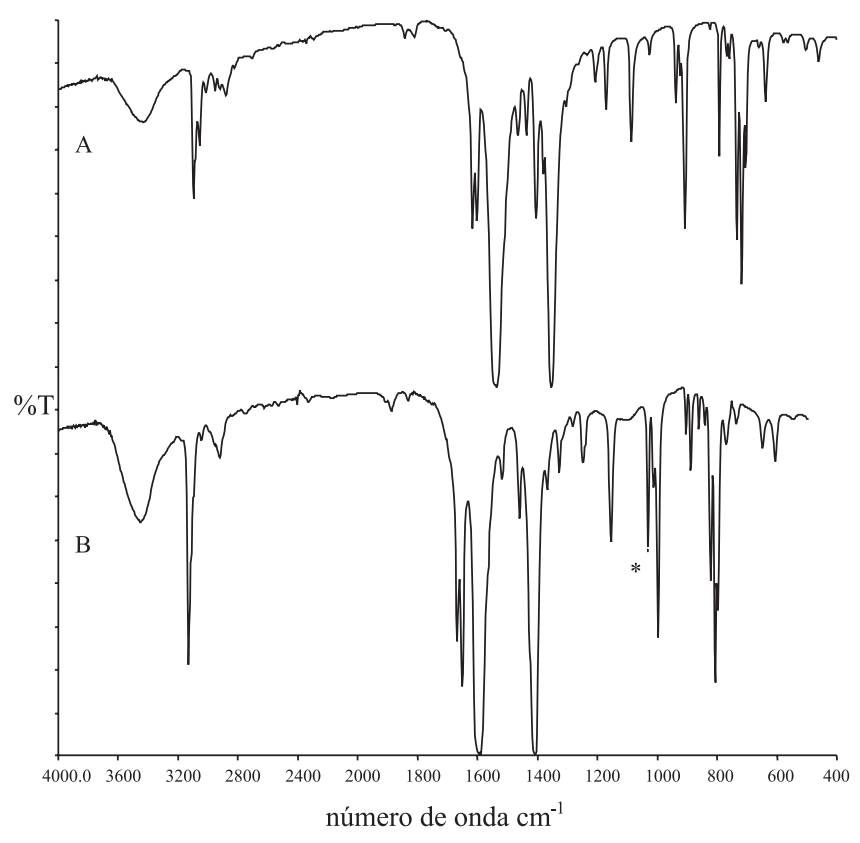

Figura 6. Espectros FT-IR obtidos por técnica de transmissão em pastilhas de $\mathrm{KBr}$, na proporção de 0,8 mg de amostra para $400 \mathrm{mg}$ de KBr: A) TNT e B)HNS

explosivos foram quantificados a partir da curva de calibração gerada. A Tabela 5 apresenta a intensidade de absorvância a $957 \mathrm{~cm}^{-1}$ de cada análise efetuada, bem como a intensidade mediana de absorvância $\left(957 \mathrm{~cm}^{-1}\right)$, o desvio padrão e o desvio relativo.

Os valores medianos de $\mathrm{A}_{957}$ (coluna 3 ) foram representados em função dos valores reais das concentrações das composições de HNS e TNT, obtidos por HPLC. A curva obtida, Figura 7, apresentou reta gerada por regressão linear segundo a Equação 4, com coeficiente de determinação $\left(\mathrm{R}^{2}\right)$ de 0,9787 .

$y=0,0024 x+0,0084$

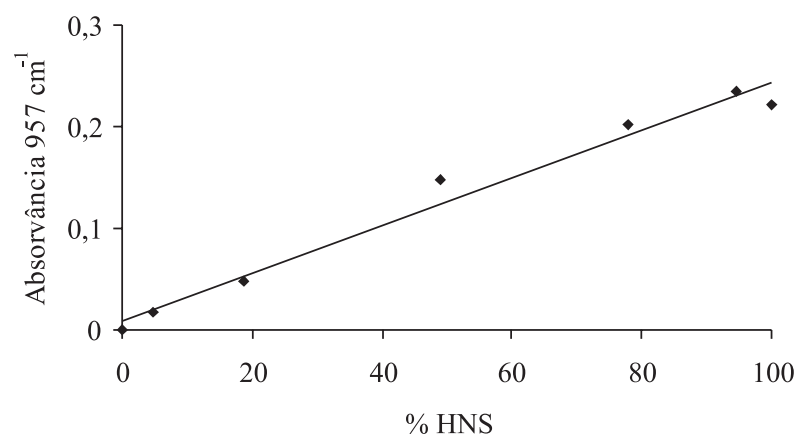

Figura 7. Gráfico de correlação entre os teores de HNS presentes nas composições e a intensidade de absorção $\left(A_{957}\right)$ do espectros FT-IR das composições analisadas

Por meio da reta média obtida e dos valores medianos das intensidades de absorção $\left(\mathrm{A}_{957}\right)$ dos espectros das composições A, B e C foram calculados os teores de HNS das composições e comparados com os valores reais obtidos por HPLC (Tabela 6).

O desvio relativo encontrado $(4,8 \%)$ está dentro da faixa aceitável para análise por FT-IR, empregando-se a técnica de pastilha. ${ }^{20}$ $\mathrm{O}$ erro apresentado entre os valores obtidos pelas duas técnicas de quantificação foi pouco significante se observada a quantidade de 
Tabela 5. Dados FT-IR obtidos com base na banda analítica de 957 $\mathrm{cm}^{-1}$

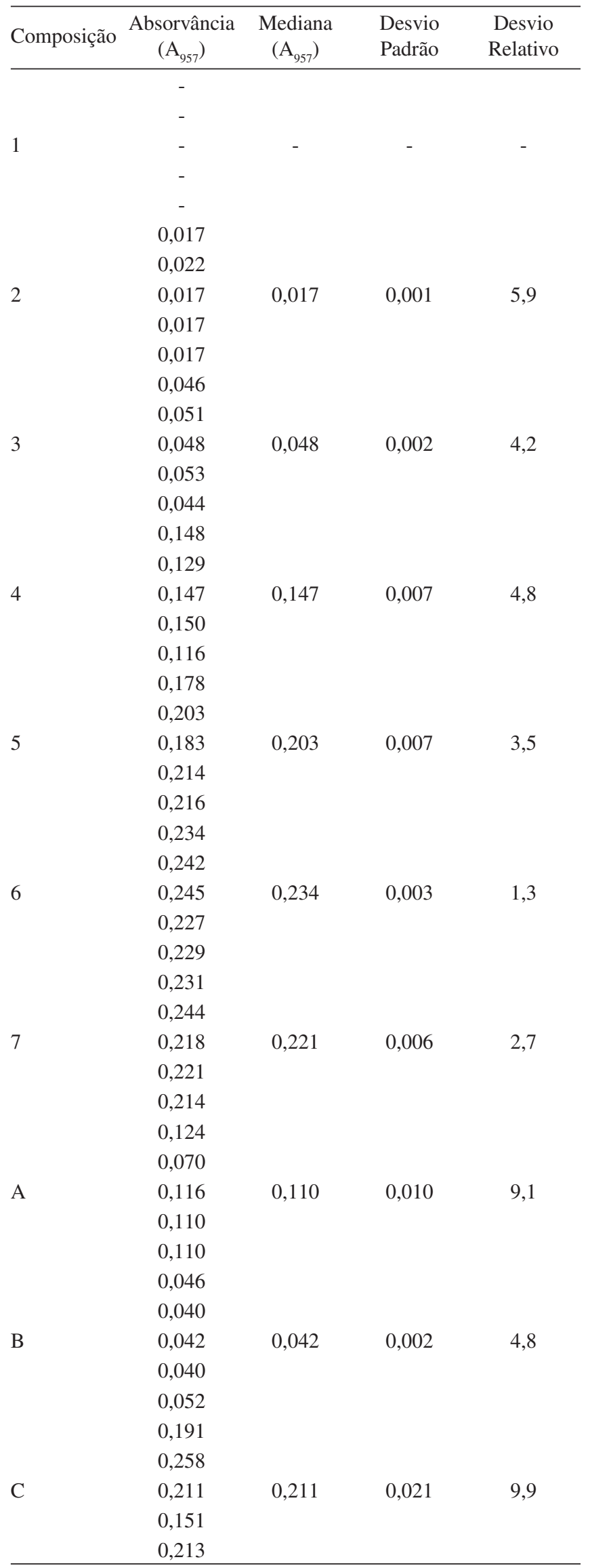

Tabela 6. Teores de HNS quantificados por FT-IR (absorvâncias medianas a $957 \mathrm{~cm}^{-1}$ ) comparados com os valores reais determinados por HPLC

\begin{tabular}{lcccc}
\hline Amostra & Mediana $\left(\mathrm{A}_{957}\right)$ & $\% \mathrm{HNS}_{\mathrm{FT}-\mathrm{IR}}$ & $\% \mathrm{HNS}_{\mathrm{HPLC}}$ & $\% \mathrm{E}$ \\
\hline A & 0,110 & 42,33 & 44,19 & 4,2 \\
$\mathrm{~B}$ & 0,042 & 14,00 & 15,06 & 7,0 \\
$\mathrm{C}$ & 0,211 & 84,42 & 89,47 & 5,6 \\
\hline
\end{tabular}

amostra empregada $(0,8 \mathrm{mg})$ e a redução no tempo e custo da análise, constituindo-se de uma opção viável para a análise de teores de HNS em composições contendo HNS e TNT.

\section{CONCLUSÕES}

A quantificação de HNS em composições contendo HNS e TNT por meio de termogravimetria é uma opção viável em função da redução no tempo e custo da análise, apesar do pequeno erro apresentado em relação aos teores determinados por HPLC, sobretudo para composições com teores mais elevados de HNS.

A determinação dos teores desses explosivos por TG representa uma metodologia rápida e que dispensa a utilização de solventes de elevado custo (DMSO e THF), como os empregados em HPLC, além de reduzir o tempo e a complexidade no preparo da amostra a ser analisada.

A metodologia baseada na análise por FT-IR utilizando como banda analítica a absorção em $957 \mathrm{~cm}^{-1}$, para a determinação do teor de HNS e TNT em composições formadas por esses explosivos, apresentou desvios relativos dentro da faixa aceitável para análise por FT-IR empregando a técnica de pastilha. Os erros apresentados entre os valores obtidos pelas duas técnicas de quantificação foram pouco significantes se observada a quantidade de amostra empregada $(0,8 \mathrm{mg})$ e a redução no tempo e custo da análise.

Face aos resultados obtidos empregando as técnicas de quantificação por TG e FT-IR conclui-se ainda que a quantificação por TG dos teores de HNS e TNT é preferível, em função de apresentar erro menor em relação aos resultados obtidos por meio de HPLC.

\section{AGRADECIMENTOS}

Aos colaboradores do Laboratório Químico da Divisão de Sistemas de Defesa (ASD) e do Laboratório de Instrumental da Divisão de Química (AQI) do Instituto de Aeronáutica e Espaço do ComandoGeral de Tecnologia Aeroespacial (CTA).

\section{REFERÊNCIAS}

1. Silva, G.; Tese de Mestrado, Instituto Tecnológico de Aeronáutica, Brasil, 2004.

2. Cumming, A. S.; Propellants, Explos., Pyrotech. 1990, 24, 46.

3. Urbansk, T.; Chemistry and Technology of Explosives, Pergamon Press: Great Britain, 1984, vol. 4.

4. Rieckmann, Th.; Völker, S.; Lichtblau, L.; Schirra, R.; Chem. Eng. Sci. 2001, 56, 1327.

5. Rieckmann, T.; Völker, S.; Lichtblau, L.; Schirra, R.; J. Anal. Appl. Pyrolysis 2001, 58, 569.

6. Silva, G.; Pinheiro, G. F. M.; Iha, K.; Dutra, R. C. L.; Takahashi, M. F. K.; Reis, T. B.; Quim. Nova 2006, 29, 681.

7. Mohan, V. K.; Field, J. E.; Combust. Flame 1984, 56, 269.

8. Harris, S. M.; Klassen, S. E.; $41^{\text {st }}$ Aerospace Sciences Meeting and Exhibit, Reno, USA, 2003.

9. Rogers, T. E.; Orpington, K.; EP.712.822, 1996.

10. Hunn, D. L.; Milam, B. T.; Havens, K. W; EP.1.722.188, 2006. 
11. Quinlin, W. T.; Thorpe, R.; Lightfoot, J. M.; U.S. 2006/0025603, 2006.

12. Neyer, B. T.; Cox, L.; Stoutenborough, T.; Tomasoski, R.; $39^{\text {th }}$ Joint Propulsion Conference and Exhibit, Huntsville, USA, 2003.

13. Silva, G.; Iha, K.; $6^{\circ}$ Simpósio de Guerra Eletrônica, São José dos Campos, Brasil, 2004.

14. Teipel, U.; Leisinger, K.; Mikonsaari, I.; Int. J. Miner. Process. 2004, $74 S$, S183.

15. Philp, D. K.; Thorpe, B. W.; J. Cryst. Growth 1976, 35, 133.

16. Silva, G.; Mattos, E. C.; Nakamura, N. M.; Iha, K.; Quim. Nova 2004,
$27,889$.

17. Andrade, J.; Iha, K.; Rocco, J. A. F. F.; Bezerra, E. M.; Iha, M. E. V. S.; Pinheiro, G. F. M. P.; Quim. Nova 2007, 30, 952.

18. Silva, G.; Tese de Doutorado, Instituto Tecnológico de Aeronáutica, Brasil, 2007.

19. Horak, M.; Vitek, A.; Interpretation and processing of vibrational spectra, John Wiley: New York, 1978.

20. Mattos, E. C.; Tese de Mestrado, Instituto Tecnológico de Aeronáutica, Brasil, 2001. 